stroke is associated with human leukocyte antigen (HLA)-B51. Ann Neurol June 1992; 31:675-677.) (Correspondence: Dr. Mintz, Department of Neurosciences, UMD-New Jersey Medical School, MSB-H506, 185 South Orange Avenue, Newark, NJ 07103.)

COMMENT. The finding of a common immunogenetic marker in children with idiopathic stroke syndrome suggests a genetic predisposition and susceptibility. The authors suggest that host factors, possibly triggered by transient viral precipitants, may contribute causally to these vascular occlusions.

Delayed onset hemiparesis and vascular thrombosis may occur approximately 6 weeks after primary varicella zoster virus infection and may explain some cases of misdiagnosed idiopathic stroke in children. (Bodensteiner JB, et al. Clinical features of vascular thrombosis following varicella. AJDC Jan 1992; 146:100-102.) The importance of this clinical entity is emphasized in an editorial (Lancet June 13, $1992 ;$ 339:1449-1450). The prognosis was good regardless of therapy and all patients recovered completely or nearly completely. The MRI was more sensitive than CT or angiography in demonstrating infarction of the basal ganglia and/or internal capsule. The delay in onset of the hemiparesis may result from the time taken for the vascular media to be infected with the virus.

\title{
CVA WITH NEUROFIBROMATOSIS TYPE I
}

A two year old developmentally delayed girl with neurofibromatosis type I and a sudden onset of left sided hemiparesis is reported from the Children's National Medical Center and George Washington University School of Medicine, Washington, D.C. There was no history of sickle cell disease or hypertension. The hemiparesis gradually improved and the patient received aspirin, $80 \mathrm{mg}$. daily. Four months later there was a left focal seizure and an acute progression of the left hemiparesis. At 5 years of age the patient presented with sudden right-sided weakness and right focal seizure. Cerebral angiography revealed bilateral distal internal carotid arterial occlusion. (Gorelick MH et al. Progressive occlusive cerebrovascular disease in a patient with neurofibromatosis type I. Clin Pediat May 1992; 31:313-315.) (Correspondence: Kenneth N. Rosenbaum, M.D., Department of Medical Genetics, Children's National Medical Center, 111 Michigan Avenue NW, Washington, D.C. 20010.)

COMMENT. Moyamoya may be seen in association with a number of systemic conditions including NF-1 or as a distinct disease entity of unclear etiology. Occlusion of the intracranial arteries is a rare complication of NF-1 and the progressive occlusion noted in this case appears to be unique.

The gene for NF-1 has been mapped to chromosome 17 and closely linked DNA markers have made accurate diagnosis by linkage analysis possible in most cases of familial NF-1. DNA testing by linkage analysis on 24 individuals with a family history of NF-1 and on 9 couples who requested testing for prenatal diagnosis was found useful as an adjunct to the clinical diagnosis 1) in children less than 6 years 
of age with incomplete clinical signs, 2) in NF-1 families for prenatal testing, and 3) when complete clinical examination is impracticle. (Hofman KJ, Boehm CD. J Pediatr March 1992; 120:394-398.)

\section{METABOLIC AND TOXIC DISORDERS}

\section{METHYLGLUTACONIC ACIDURIA}

Two siblings with 3-methylglutaconic aciduria with normal enzyme activity and neurological abnormalities are reported from the Department of Pediatrics, Beilinson Medical Center, Petah Tiqva, Sackler School of Medicine, Tel Aviv University, Israel. Patient 1, an 11 month old infant was referred for evaluation of deterioration of motor development beginning at 5 months of age. There was an arrest of weight gain and head circumference growth. He was restless and had choreoathetoid movements of hands, head and mouth, deep tendon reflexes were hyperactive, plantar responses were extensor and optic discs atrophied. At 2 years of age he sat with support, crawled and walked with help. MRI and CT showed prominent frontal lobe atrophy. Urinary organic acid analysis showed a prominent peak of 3methylglutaconic and 3-methylglucrylic acid. Patient 2 , the 14 year old sister of patient 1 , developed ataxia of gait at age 2 years, and optic atrophy at 8 years of age. Examination showed variable muscle tone with brisk tendon reflexes and extensor plantar responses. Choreoathetoid movements were prominent in the upper limbs, head and mouth and funduscopic examine showed prominent optic atrophy. Her IQ on the WISC was 87. CT and MRI showed generalized brain atrophy. Urinary organic acid excretion showed a prominent peak of methylglutaconic and methylglutaric acids. The patients represent a new clinical variant of the methylglutaconic aciduria syndrome with a relatively favorable prognosis. (Zeharia A, Weitz $R$ et al. 3-

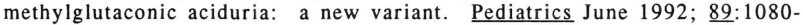
1082.) (Reprints: Avraham Zeharia, M.D., Department of Pediatrics B, Beilinson Medical Center, Petah Tiqva 49100, Israel.)

COMMENT. 3-Methylglutaconic aciduria is the hydrolysis product of 3-methylglutaconyl coenzyme and an intermediate in the degradation pathway of leucine. Two syndromes are described - one with deficient methylglutaconyl coenzyme $A$ and the other with normal enzyme activity, but prominent neurological deterioration. The present case reports were unique in the relatively normal cognitive and intellectual development and the relatively mild neurological manifestations. The boy had demonstrated developmental improvement in his second year of life and his sister developed well with normal school performance.

\section{COCAINE ADDICTION AND EEG IN INFANTS}

Thirty-five consecutive infants of cocaine-addicted mothers hospitalized for a comprehensive health assessment and 51 healthy, age-matched infants were studied with electroencephalography at the Children's Hospital of Philadelphia, PA. No definite EEG seizures were recorded in any of the patients. In infants of cocaine-addicted mothers there was a tendency for 